\title{
Observation of low frequency Kelvin waves in the mesosphere
}

\author{
Anne K. Smith \\ Atmospheric Chemistry Division, National Center for Atmospheric Research, Boulder, CO 80307, U.S.A.
}

(Received August 4, 1998; Revised February 12, 1999; Accepted February 12, 1999)

\begin{abstract}
This paper presents evidence for a quasi-stationary Kelvin wave of zonal wavenumber 1 that is forced in situ in the upper mesosphere and propagates upward from there. Although large scale quasi-stationary longitudinal variations are common in the mesopause equatorial winds, these variations often do not have a structure associated with a vertically propagating mode. However, during the periods of easterly winds that occur near the equinoxes in association with the mesopause semi-annual oscillation (MSAO), a Kelvin wave propagates away from the presumed altitude of forcing. The momentum transport by the Kelvin wave is small and does not make a significant contribution to the SAO wind evolution.
\end{abstract}

\section{Introduction}

A stationary wavenumber 1 zonal asymmetry is a persistent feature of the zonal winds measured by the High Resolution Doppler Imager (HRDI) at the equatorial mesosphere (Smith, 1997). This asymmetry was shown to have a narrow vertical and meridional extent, and to occur during both the easterly and westerly phases of the mesospheric semi-annual oscillation (MSAO). The wave 1 amplitude is maximum around $80 \mathrm{~km}$ during the easterly phase of the MSAO; the longitudinal phase of the perturbation is opposite during the easterly and westerly phases of the MSAO, but is the same from year to year. The limited extent of the wave signal in the meridional-vertical plane suggests that it is generated by a persistent localized momentum source, such as would occur with large scale longitudinal variations in the generation or transmission of small scale waves. Dissipation of gravity waves that propagate from the troposphere is believed to be a dominant source of momentum in the upper mesosphere. In the equatorial region, the seasonally varying zonal winds of the mesopause semi-annual oscillation (Lieberman et al., 1993; Garcia et al., 1997) may be driven by gravity waves (Dunkerton, 1982) or diurnally forced medium scale waves (Sassi and Garcia, 1997).

A localized deposition of momentum into the atmosphere can change the local winds and can drive a three-dimensional circulation that maintains the thermal and angular momentum conservation requirements. In some circumstances, the localized momentum source will give rise to motions that can propagate, and the momentum will be further redistributed. This paper presents observational evidence that a propagating wave, in this case a vertically propagating Kelvin wave, is generated in the upper mesosphere during the easterly phase of the MSAO. The wave is confined to a narrow altitude range determined by the depth of the MSAO easterly winds.

Copy right(c) The Society of Geomagnetism and Earth, Planetary and Space Sciences (SGEPSS); The Seismological Society of Japan; The Volcanological Society of Japan; The Geodetic Society of Japan; The Japanese Society for Planetary Sciences.

\section{Data}

Direct wind measurements by the HRDI instrument on the UARS satellite have been particularly useful in the tropics where the geostrophic relation used to derive wind from temperature is not reliable. The HRDI data and validation are described by Hays et al. (1993), Burrage et al. (1995), and Ortland et al. (1996). The present study uses mesospheric HRDI wind data archived as level 3at, version 10, ranging from 55 to $114 \mathrm{~km}$.

The $\mathrm{O}_{2}\left({ }^{1} \Sigma\right)$ emissions that were detected by HRDI for deriving the horizontal winds also contain information about the mesospheric temperature. The dependence of emission signal on temperature and on volume emission rate (VER) varies for different lines detected by HRDI; this information can be used to solve simultaneously for the temperature and VER (Ortland et al., 1998). The estimated error is about $7 \mathrm{~K}$ in the region from 80 to $100 \mathrm{~km}$. Below about $80 \mathrm{~km}$, the error increases because the temperature inversion is more heavily dependent on a priori information. See Ortland et al. (1998) for a description of the data product and error analysis. Only monthly zonal mean temperatures are used in this paper; the temperature uncertainties are too large for the confirmation of the wave signatures described in the wind observations.

For all wind analyses in this paper, profiles are binned in latitude and averaged in time to reduce noise and to isolate the steady features. In order to investigate waves in the HRDI data, the wind variations due to tides must be separated from other wave fields. UARS precesses about 20 minutes per day, so in a given day (for viewing consistently towards the same side of the spacecraft), all the HRDI data are taken at approximately the same local time. The longitudinal average of a day's data therefore contains the true zonal mean field and the contributions from all the migrating tides. When the longitudinal average is removed, the perturbation may contain non-migrating tides, planetary waves and variability due to other sources. During some days beginning in 1994, HRDI viewed on opposite sides of the spacecraft during alternate orbits, thus increasing the local time coverage (about 2 hours 


$$
4 / 29 / 93-5 / 27 / 93 \quad 10 S-10 N
$$
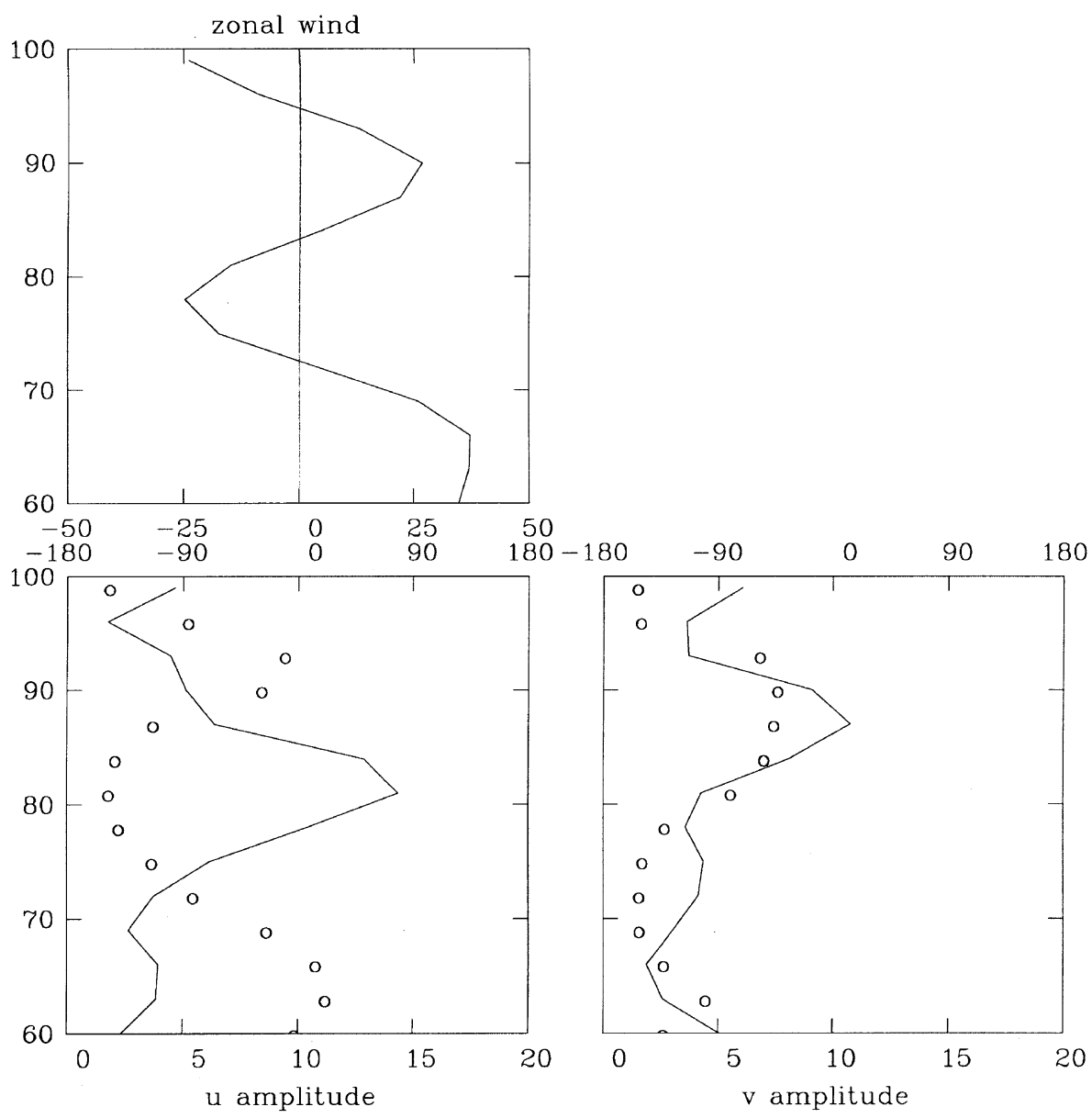

Fig. 1. Vertical profiles averaged over the latitudes $10^{\circ} \mathrm{S}$ to $10^{\circ} \mathrm{N}$ for 29 April to 27 May, 1993. Top panel is the longitudinal average zonal wind (units $\mathrm{m} \mathrm{s}^{-1}$ ), bottom left panel is the zonal wind wavenumber 1 amplitude (solid line; units $\mathrm{m} \mathrm{s} \mathrm{s}^{-1}$; scale at bottom of frame) and phase (open circles; units degrees; scale at top of frame) and the bottom right panel is meridional wind wavenumber 1 amplitude and phase.

difference at the equator). This mode is taken into account when determining the perturbation wind by removing the mean for each viewing orientation separately.

Persistent nonmigrating tides will be sampled at a different phase on each day and will appear as temporal variations with an apparent period equal to the precession of the UARS satellite (36 days) or a fraction of it. Any systematic longitudinal perturbation with periods longer than this must have another cause. Most of the results presented in this paper were averaged over one full yaw cycle, which is less than or equal to the precession period, so nonmigrating tidal influences are minimized. The total (non-perturbation) zonal winds are also shown in this paper. Some contamination by the migrating tides still exists since HRDI measures only during daytime, although this has been reduced by averaging in time (Forbes et al., 1997). Due to the long precession period of the UARS satellite, analysis and removal of the migrating diurnal tide would have also removed part of the true variation of the zonal mean wind.

\section{Observed Wave Characteristics}

The analytical structure of the Kelvin wave on an equatorial $\beta$-plane was described by Holton and Lindzen (1968).
The idealized Kelvin wave in an atmosphere with no meridional shear has a particularly simple form since the meridional wind associated with the wave is identically zero. For a slowly varying background winds, the structure can also be generalized for a background atmosphere with vertical (Holton, 1970; Lindzen, 1971) or meridional (Boyd, 1978a,b) shear. Numerous observations of Kelvin waves in the middle atmosphere confirm that the theoretical structure does a good job of describing the observed waves. The latitudinal structure, particularly the symmetry across the equator and equatorial trapping, are normally used to isolate the Kelvin wave signals in zonal wind, geopotential, temperature or trace chemical constituents.

\subsection{Wave structure in HRDI zonal wind}

Figure 1 shows vertical profiles of three different monthly mean quantities averaged over the latitude band $10^{\circ} \mathrm{S}$ to $10^{\circ} \mathrm{N}$ for the period 27 April through 28 May, 1993 (a complete yaw cycle). The period includes 13 days with mesospheric wind data. The panels give the average wind speed, the amplitude and phase of the monthly mean perturbation zonal wind, and the amplitude and phase of the monthly mean perturbation meridional wind. At the beginning of this period there were 
strong easterly winds at the equator, with peak velocity of about $-90 \mathrm{~m} / \mathrm{s}$ at $80-85 \mathrm{~km}$; during the period the magnitude of the easterlies decreased and the altitude of the maximum moved down until, by the end of the month, the zonal average easterly winds in the upper mesosphere were confined to a narrow region around $80 \mathrm{~km}$. The wave analysis of zonal wind indicates a strong wave 1 pattern with peak amplitude of about $14 \mathrm{~m} / \mathrm{s}$ slightly above the altitude of the peak mean easterly wind. At this altitude, the meridional wind has only a small amplitude, with a local minimum coinciding with the peak in zonal wind amplitude.

The phase of the zonal wind wave tilts eastward with height above about $80-85 \mathrm{~km}$, as expected for an upward propagating Kelvin wave. The phase below $80 \mathrm{~km}$, however, tilts in the other direction. Although the westward phase tilt could indicate that the wave is propagating downward, it could also be interference with the non-migrating tides that are frequently seen at this altitude (Talaat and Lieberman, 1998).

The HRDI data for each latitude bin were analyzed in a similar way and combined to give the meridional structure. Figure 2 shows the wavenumber 1 zonal wind amplitude as a function of latitude (equatorward of $40^{\circ} \mathrm{N}$ and $\mathrm{S}$ ) and altitude $(60$ to $100 \mathrm{~km}$ ) for the April-May 1993 yaw cycle. The feature that was described in Fig. 1 is evident between 80 and $85 \mathrm{~km}$, extending northward and southward from the equator. Note that the region of significant wave amplitude is not symmetric about the equator but is shifted toward the Southern Hemisphere, and that the wave amplitude increases with altitude over a $10 \mathrm{~km}$ range $(70-80 \mathrm{~km})$ and then decreases abruptly between 80 and $87 \mathrm{~km}$.

Tropical winds and wave amplitudes for a second period (October, 1994) are shown in Fig. 3. During this period, as during the April-May 1993 period presented above, the

wave $14 / 29-5 / 27$

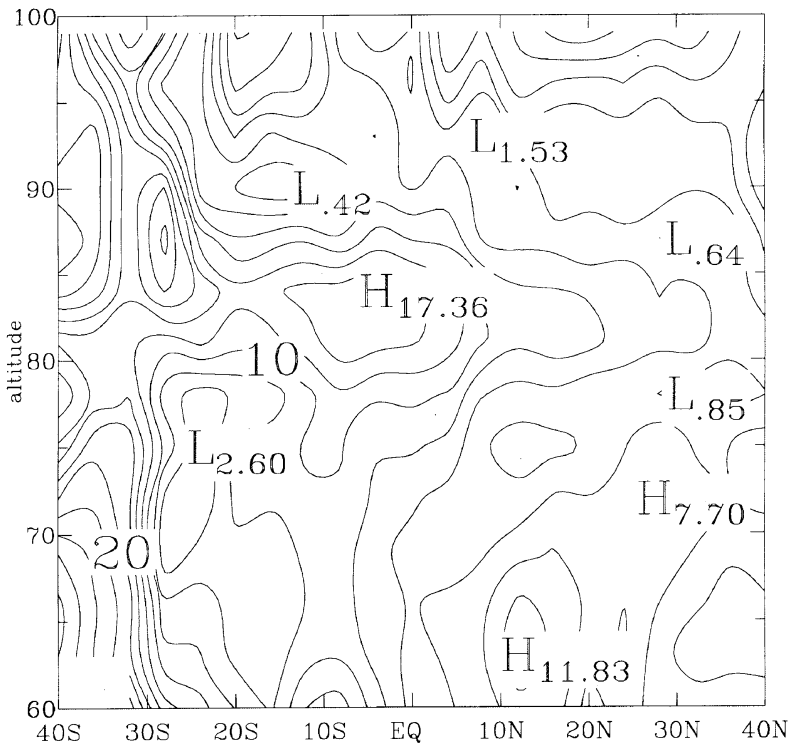

Fig. 2. Latitude $\times$ altitude cross-section of the zonal wind wavenumber 1 amplitude for 29 April to 27 May, 1993. easterly winds of the SAO have diminished from strong to more moderate speeds, and are still decreasing. There are a number of features in common with the April-May case shown above. The wave amplitude maximum occurs slightly above the altitude of maximum easterly winds. The phase tilts eastward with height over a limited range $(82-92 \mathrm{~km})$ although the eastward phase tilt is less pronounced than in the 1993 case. As in April-May 1993, there is westward phase tilt below the region of maximum amplitude. Amplitude of the meridional wind is small. A latitude altitude cross-section of the zonal wind wave 1 amplitude is shown in Fig. 4. As in the 1993 results in Fig. 2, the amplitude around $80-85 \mathrm{~km}$ is significant at the equator but the maximum is shifted toward the fall (in this case, northern) hemisphere.

Characteristics that can be used to identify a Kelvin wave are 1) the meridional wind speed is negligible; 2) the wave propagates eastward relative to the background wind; 3 ) the phase tilts eastward with increasing altitude for an upward propagating wave; 4) the zonal and meridional wavenumbers are related to the frequency through a dispersion relation; and 5) the wave is centered on the equator and equatorially trapped with a width dependent on the zonal wavenumber and frequency.

Both waves satisfy the first criterion (small meridional wind). Cross-sections of the meridional wind wavenumber 1 amplitude (not shown) do not give any indication of a coherent wave present at the equator. The observed waves in zonal wind also satisfy the second criterion (eastward propagation), since the wave is stationary and occurs in an easterly (westward) flow. The eastward vertical phase tilt was clearly seen for both of these waves, and was the feature that initially indicated that a Kelvin wave might be present.

The dispersion relation gives the vertical wavenumber if the frequency and zonal wavenumber are known. For an idealized stationary Kelvin wave

$$
m^{2}=\frac{N^{2} k^{2}}{\omega^{2}}\left[1+\frac{\bar{u}_{z} \omega}{H N^{2} k}\right] \approx \frac{N^{2}}{\bar{u}^{2}}\left[1-\frac{\bar{u}_{z} \bar{u}}{H N^{2}}\right] \approx \frac{N^{2}}{\bar{u}^{2}}
$$

or

$$
L_{z}=2 \pi \frac{\bar{u}}{N}
$$

where $k$ and $m$ are the zonal and vertical wavenumbers, $N$ is the Brunt-Väisälä frequency, $\omega$ is the wave frequency, $H$ is the atmospheric scale height and $\bar{u}$ is the mean zonal wind speed in the equatorial region. The vertical wavelength $L_{z}$ (where $L_{z}=2 \pi / \mathrm{m}$ ) is proportional to the wind speed. For winds of 30 and $60 \mathrm{~m} / \mathrm{s}$, the predicted vertical wavelengths are 9 and $18 \mathrm{~km}$, respectively. It is difficult to determine a precise vertical wavelength from the HRDI wind observations because of the limited vertical resolution $(3 \mathrm{~km})$ and the narrow range of height over which the wave is present. Also, the field of view of the instrument and the smoothing incorporated in the inversion of the data limit the vertical wavelengths that can be resolved. Taking individual vertical points, the apparent wavelength for the 1993 case is as short as $10-12 \mathrm{~km}$ over at least part of the range where it is growing with altitude.

The fifth condition (symmetric, equatorially trapped) is not met very well by the observed waves; the maximum wave amplitude in zonal wind is shifted off the equator, in both 


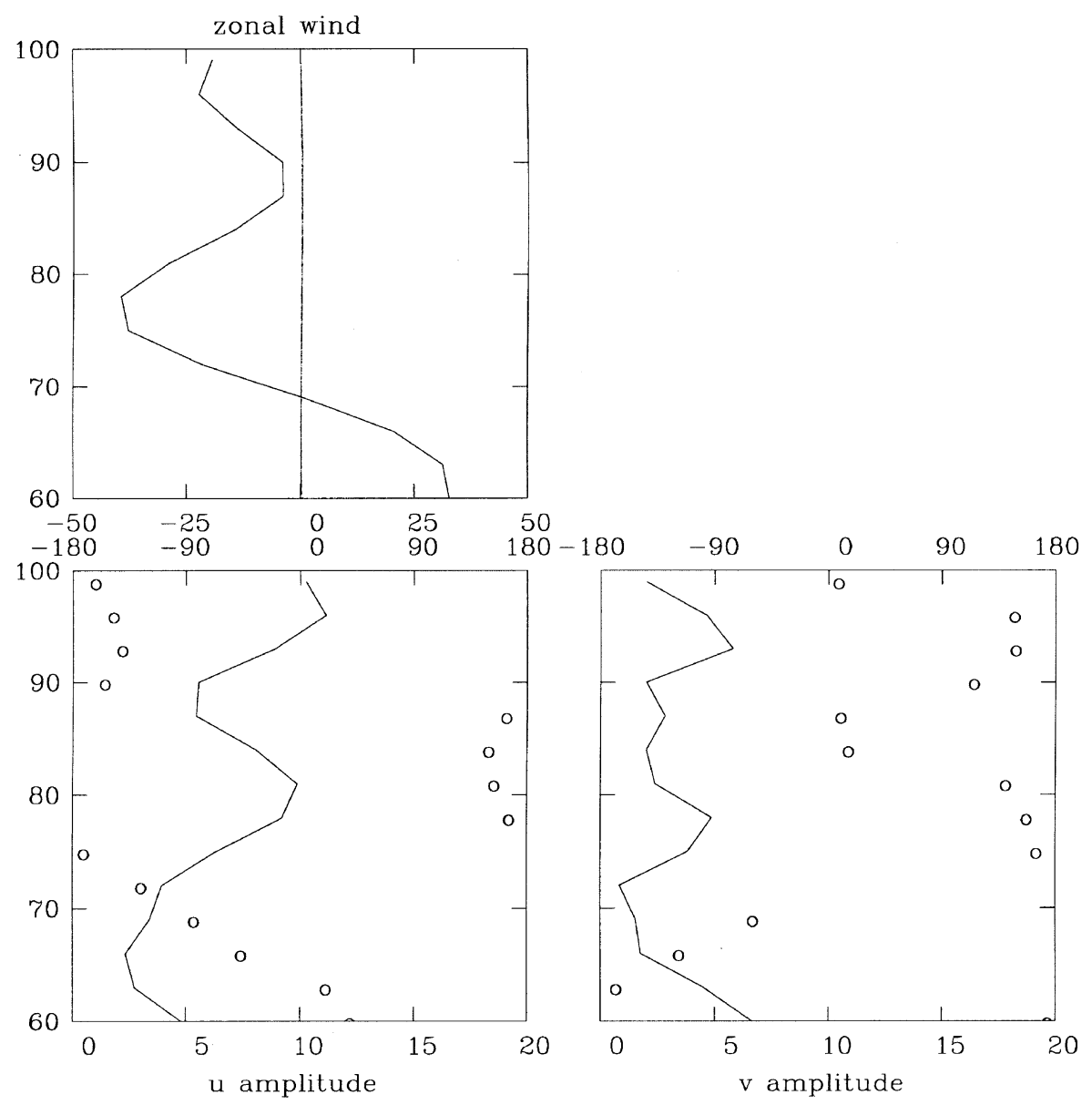

Fig. 3. As in Fig. 1, but for October, 1994.

cases toward the fall hemisphere. A certain amount of asymmetry is expected in the presence of a meridional wind shear (Boyd, 1978a,b). However, Boyd's analysis indicates that the asymmetric component of the Kelvin wave eddy zonal wind should be a small fraction of the symmetric component. For linear meridional shear, the ratio of the asymmetric $\left(u_{1}\right)$ to symmetric $\left(u_{0}\right)$ components is given by

$$
\frac{u_{1}}{u_{0}}=-\frac{k \omega_{0}}{\beta}\left[\frac{\omega(y)-\omega_{0}}{\omega_{0}}\right] \approx \frac{k^{2}}{\beta} \bar{u}_{y} \cdot y
$$

where $\beta$ is the gradient of Coriolis torque, $y$ is the horizontal distance from the equator, and we have used the relations for stationary waves: $\omega_{0}=-k \bar{u}_{0}$ and $\omega(y) \approx \omega_{0}-k \bar{u}_{y}$. $y$. For easterly winds $(\bar{u}<0)$, Eq. (1) predicts that the wave amplitude should be larger ( $u_{0}$ and $u_{1}$ are in phase) in the hemisphere with weaker easterly winds. But even for the large and narrowly confined zonal wind maxima of the easterly phase of the MSAO, the predicted asymmetry is quite small (a few percent).

From Boyd's analysis, the meridional structure of a Kelvin wave including the asymmetry for a linear shear can be calculated from

$$
u(y)=\left(u_{0}+u_{1}\right) \exp \left[\frac{-\beta k y^{2}}{2(\omega-k \bar{u})}\right]
$$

$$
\approx u_{0}\left(1+\frac{k^{2}}{\beta} \bar{u}_{y} y\right) \exp \left[\frac{\beta y^{2}}{2 \bar{u}}\right] .
$$

The e-folding widths for equatorial mean winds of -30 and $-60 \mathrm{~m} / \mathrm{s}$ are about $15^{\circ}$ and $20^{\circ}$, respectively. Figures 5 and 6 show the observed variation of zonal wind wavenumber 1 amplitude with latitude as well as the variation predicted by Eq. (2). Both have been normalized to one at the equator. The observed amplitudes conform quite well to the theoretical variations in the spring hemisphere but are significantly larger and more irregular than predicted in the fall hemisphere. It is evident that the asymmetries in the observations cannot be explained as the modification of a classical Kelvin wave by meridional shear of the background flow.

Overall, the waves observed in HRDI winds in April-May, 1993 and October, 1994 conform well to a theoretical Kelvin wave, with the primary discrepancy being the structure of the wave in the meridional dimension. In particular, the wave amplitude maximum in the autumn hemisphere is not predicted. Possible explanations for the cross-equatorial asymmetry include that the forcing that excites the wave is located off the equator or that the wave is preferentially damped in one hemisphere. Forcing of the Kelvin wave will be discussed in Section 4, but there is no tangible evidence that can be used to identify the forcing mechanism, asymmet- 
1994

u wave $110 / 1-10 / 31$

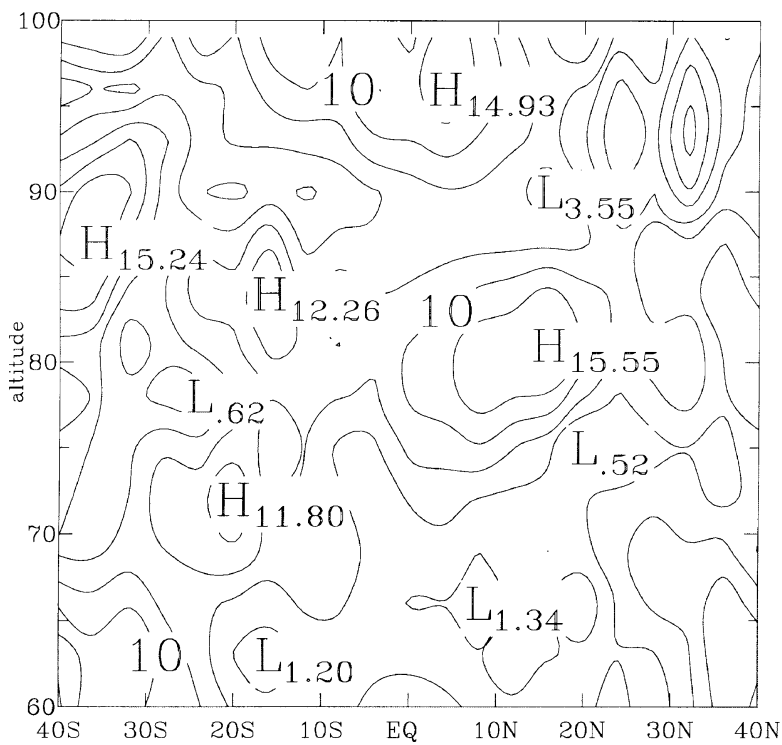

Fig. 4. Latitude $\times$ altitude cross-section of the zonal wind wavenumber 1 amplitude for October, 1994

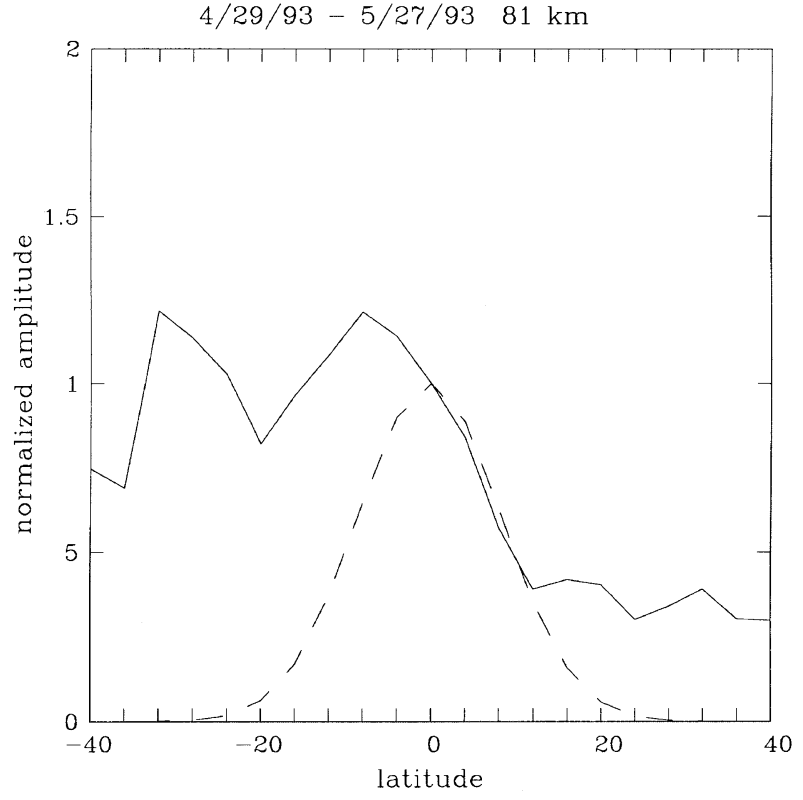

Fig. 5. Solid curve is the zonal wind wavenumber 1 amplitude at $81 \mathrm{~km}$ for 29 April to 27 May, 1993, normalized to one at the equator. Dashed curve is the meridional structure of an idealized stationary Kelvin wave for a zonal wind speed of $-25 \mathrm{~m} / \mathrm{s}$.

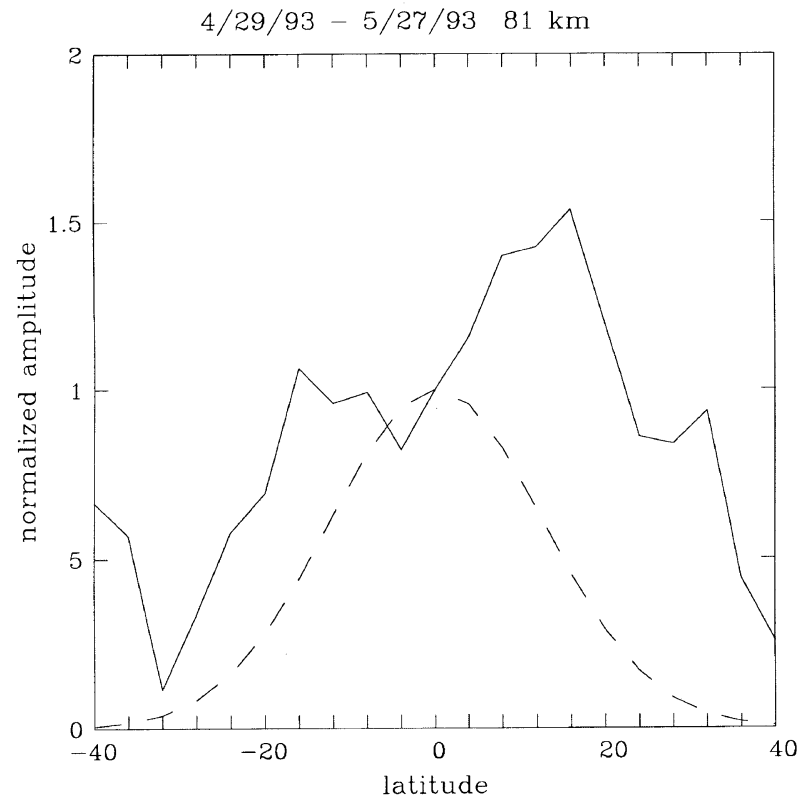

Fig. 6. Solid curve is the zonal wind wavenumber 1 amplitude at $81 \mathrm{~km}$ for October, 1994, normalized to one at the equator. Dashed curve is the meridional structure of an idealized stationary Kelvin wave for a zonal wind speed of $-50 \mathrm{~m} / \mathrm{s}$. data by Lieberman and Riggin (1997) in the mesospherelower thermosphere region (above $\sim 90 \mathrm{~km}$ ) appear also to have been generated in situ.

\subsection{Wave structure in the presence of gravity wave drag}

The mean winds in the upper mesosphere are believed to be strongly influenced by breaking and dissipation of gravity waves that propagate from the troposphere. Gravity wave breaking would also influence the large scale zonal wind variations associated with the Kelvin wave. Consider the linearized eddy zonal momentum equation for a Kelvin wave (meridional winds set to zero) with an additional contribution $F_{\mathrm{g}}$ for the momentum source generated by gravity wave breaking.

$$
\left(\frac{\partial}{\partial t}+\bar{u} \frac{\partial}{\partial x}\right) u^{\prime}+\bar{u}_{z} w^{\prime}+\Phi_{x}=F_{\mathrm{g}}
$$


where $\Phi_{x}$ is the zonal gradient of geopotential. For representation of the momentum source, we use the formula of Lindzen (1981)

$$
F_{\mathrm{g}}=-\int_{i} A\left(u-c_{i}\right)^{3}
$$

where

$$
A=\frac{k_{\mathrm{g}}}{2 N}\left[\frac{1}{H}-\frac{3 u_{z}}{(u-c)}\right]
$$

and $u$ is the background wind. Linearizing (4), the eddy component of $F_{\mathrm{g}}$ is given by

$$
\begin{aligned}
F_{\mathrm{g}}= & -u^{\prime}\left\{\int_{i} \frac{k_{\mathrm{g}}}{2 N}\left[\frac{3}{H}-\frac{6 \bar{u}_{z}}{\left(u-c_{i}\right)}\right]\left(u-c_{i}\right)^{2}\right\} \\
& +3 u_{z}^{\prime} \int_{i} \frac{k_{\mathrm{g}}}{2 N}\left(u-c_{i}\right)^{2} .
\end{aligned}
$$

Equation (5) also uses the assumption that the flux of gravity waves is similar at all longitudes, so that $\int_{i} c_{i}$ has no significant wave component. The first term on the right has the form of a damping coefficient on $u^{\prime}$, while the second is $1 / 4$ cycle out of phase with the damping and will tend to move the wave phase downward.

The eddy zonal momentum equation for a stationary wavenumber 1 can be written

$$
(i k \bar{u}+\alpha) u^{\prime}+\bar{u}_{z} w^{\prime}+\Phi_{x}=0 .
$$

The real part of $\alpha$, which is the damping coefficient from (5), is then

$$
\alpha=3 \int_{i} \frac{k_{\mathrm{g}}}{2 N}\left[\frac{1}{H}-\frac{2 \bar{u}_{z}}{\left(\bar{u}-c_{i}\right)}\right]\left(\bar{u}-c_{i}\right)^{2} .
$$

Some of the fields needed to determine $\alpha$ are known from the measurements. In particular, the mesospheric zonal mean wind is measured by HRDI and the Brunt-Väisälä frequency can be computed from the observed (HRDI) temperature. The distribution of phase speeds $c_{i}$ that can occur at a given level is constrained by the wind profile from the tropopause to the level in question. Zonal winds and temperatures for the stratosphere are determined from NCEP (National Center for Environmental Prediction) data using the method of Randel (1987). Missing fields are the gravity wave zonal wavenumber $k_{\mathrm{g}}$, the range of phase speeds $c$ at the tropopause, and the amplitudes or breaking levels of the gravity waves. To obtain an estimate of the structure of the damping coefficient, we use the gravity wave drag parameterization that has been used in numerous middle atmosphere models beginning with Holton (1983). In the present case, we compute the drag assuming there is a range of 11 discrete phase speeds at the tropopause ranging from $-50 \mathrm{~m} / \mathrm{s}$ to $50 \mathrm{~m} / \mathrm{s}$. For each phase speed, waves are present at three different initial amplitudes (the parameter $\tilde{u}$ in the formulation of Holton). The three values of $\tilde{u}$ are the same for each frequency, and were chosen to give a range of breaking heights from the lower mesosphere to the lower thermosphere for gravity waves that do not encounter a critical layer.

Figures 7 and 8 show $\alpha$ calculated from the parameterization for the two periods considered above. It is clear that $\alpha$

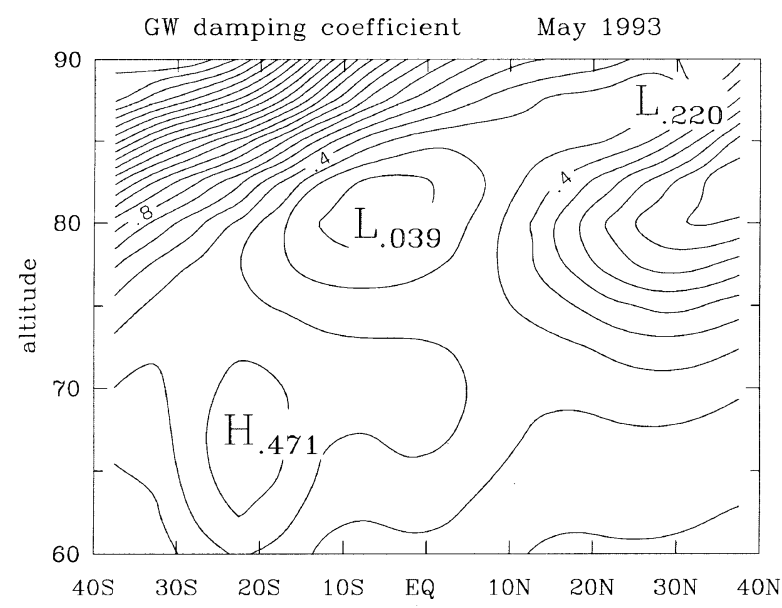

Fig. 7. Damping rate (m/s/day) due to parameterized gravity wave breaking, using the observed winds and temperatures for the period 29 April to 27 May, 1993.

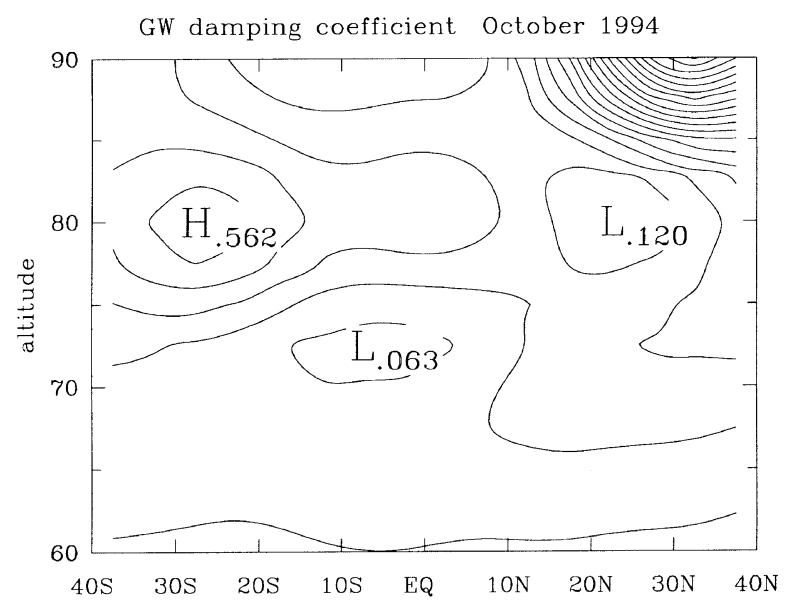

Fig. 8. Damping rate ( $\mathrm{m} / \mathrm{s} /$ day) due to parameterized gravity wave breaking, using the observed winds and temperatures for October, 1994.

is positive (damping) everywhere but shows significant variability. Smallest values of $\alpha$ occur where the vertical gradient is strong and the zonal wind speed is weak. Comparison of Fig. 7 with Fig. 2 and of Fig. 8 with Fig. 4 shows that the minimum damping (indicated by "L" on Figs. 7 and 8 ) is located near $80-85 \mathrm{~km}$ on the autumn side of the equator, approximately colocated with the maximum of zonal wavenumber 1 .

The close correspondence between the minimum in the calculated gravity wave drag and the maximum in the planetary wave amplitude suggests that this may contribute to the variations with longitude. Although the absolute magnitude of the damping coefficient cannot be determined from the available data (and is normally tuned in models to give the observed seasonal evolution), the structure in the tropical mesosphere is largely a result of the observed mean wind, not only locally but also extending down to the troposphere, and not of the adjustable parameters. 


\section{Origin of the Kelvin Wave}

In this section, we summarize observational evidence that is available and is believed to be relevant to the question of what generated the Kelvin wave described above. Unfortunately, observations are not sufficient to confirm the hypothesis.

Since Kelvin waves can propagate upward only in easterly winds, it is not possible that the observed waves propagated from the troposphere. Furthermore, they could not have propagated even from the stratosphere or lower mesosphere since, during the periods they were observed, easterly winds at the equator existed only above about $70 \mathrm{~km}$. Since the waves are stationary with respect to the ground, it is reasonable to assume that their origin is related to fixed geographical features.

In addition to the Kelvin wave structure described in this paper, a wavenumber 1 asymmetry in the zonal wind that does not appear to be a Kelvin wave is seen much more often, and has its largest amplitude during the period of strongest winds near the mesopause. This persistent tropical asymmetry was described by Smith (1997); it was hypothesized to originate from large scale longitudinal variations in the momentum deposition by small scale waves. These variations could result from regional differences in the generation of gravity waves, such as associated with regional centers of instability or convective activity, or from filtering of gravity waves by planetary scale wind variations in the troposphere or stratosphere. Examination of the tropospheric and stratospheric winds calculated from NMC analyses (surface to $1 \mathrm{mb}$ ) and measured by HRDI (25-40 km) turned up no evidence for filtering, although the wind measurements in the tropical atmosphere may not be reliable enough to isolate longitudinal variations.

The Kelvin wave identified in this paper occurs over certain periods during the easterly phase of the mesopause SAO, particularly during times when the mean zonal wind speed is decreasing. Its relationship to the persistent asymmetries in the MSAO may be relevant to its origin. The phase of the persistent asymmetry gives maximum (i.e. minimum easterly wind speed) in the longitude region around $120^{\circ} \mathrm{E}-180^{\circ} \mathrm{E}$. Referring to Figs. 1 and 3, we see that the Kelvin wave phase at its maximum is $150^{\circ} \mathrm{W}$ (April-May, 1993) and $150^{\circ} \mathrm{E}$ (October, 1994). In other words, near its maximum amplitude the Kelvin wave is close to being in phase with the persistent asymmetry. Therefore, it is conceivable that the momentum forcing that generates the persistent asymmetry is sometimes able to force a coherent vertically-propagating Kelvin wave. Variations of the forcing with latitude may also account for or contribute to the observed asymmetry about the equator.

\section{Impact on the Mean Flow}

Kelvin waves give an upward flux of westerly momentum. Where they dissipate they will damp the easterly winds through which they have propagated. Here we estimate the momentum flux convergence in order to determine whether the redistribution of momentum by the Kelvin wave could have an impact on the evolution of the MSAO winds.

From the eddy continuity equation

$$
\frac{\partial u}{\partial x}+\frac{1}{\rho} \frac{\partial(\rho w)}{\partial z}=0
$$

and for a stationary wave,

$$
w^{\prime}=\frac{-i k u}{\left(i m-\frac{1}{2 H}\right)} \approx-\frac{k u}{m}
$$

where $m$ is the vertical wavenumber, determined from the vertical structure of the observed wave. The EP flux divergence can be estimated by

$$
\nabla \cdot F=-\frac{\partial}{\partial z} \rho \overline{u^{\prime} w^{\prime}}=\frac{\partial}{\partial z}\left(\frac{\rho k \overline{u^{\prime 2}}}{m}\right) .
$$

For the two cases presented, the EP flux divergence gives a zonal momentum forcing of less than $1 \mathrm{~m} / \mathrm{s} /$ day. This drag is small compared with estimated drag from breaking gravity waves in the upper mesosphere. Therefore, we conclude that the presence of the Kelvin wave has no significant impact on the evolution of the mesopause SAO.

\section{Conclusions}

A persistent wave 1 pattern in tropical upper mesospheric winds was observed in HRDI data during several periods near equinoxes. The wave conforms in many ways to a theoretical Kelvin wave. The Kelvin wave studied here is unusual because it is stationary. For a stationary wave, the vertical wavelength is proportional to the wind speed; a wavelength large enough to be detected and to persist against thermal damping will occur only with easterly wind speeds in the range of $20 \mathrm{~m} / \mathrm{s}$ or greater.

The wave conforms to a theoretically predicted Kelvin wave in most aspects, including having very weak meridional wind, approximately satisfying the dispersion relation, and being confined to the equatorial region. The primary deviation from the predicted structure is the meridional structure. In particular, wave amplitude does not peak at the equator but is offset toward the fall hemisphere. The amplitude maximum coincides closely with the minimum in the effective gravity wave damping rate deduced from the Lindzen parameterization. This suggests that the wave maximum may occur where it does because that is the region of weakest damping, and provides a plausible explanation for the offset of the wave maximum from the equator.

The generation mechanism for the wave is not known. Planetary wave activity could result from zonally asymmetric momentum deposition by smaller scale waves. This momentum deposition would need to be continuous to maintain the wave in the face of strong damping by breaking gravity waves and dissipation due to turbulent diffusion.

Acknowledgments. I would like to thank C. Cavanaugh and F. Wu for help with reading the HRDI level 3 data. This research was supported by the NASA UARS Guest Investigator Program under grant S-12896F. The National Center for Atmospheric Research is supported by the National Science Foundation.

\section{References}

Boyd, J. P., The effects of latitudinal shear on equatorial waves. Part I: theory and methods, J. Atmos. Sci., 35, 2236-2258, 1978a.

Boyd, J. P., The effects of latitudinal shear on equatorial waves. Part II: applications to the atmosphere, J. Atmos. Sci., 35, 2259-2267, 1978 b.

Burrage, M. D., W. R. Skinner, D. A. Gell, P. B. Hays, A. R. Marshall, D. A. Ortland, A. H. Manson, S. J. Franke, D. C. Fritts, P. Hoffman, C. McLandress, R. Niciejewski, F. J. Schmidlin, G. G. Shepherd, W 
Singer, T. Tsuda, and R. A. Vincent, Validation of mesosphere and lower thermosphere winds from the High Resolution Doppler Imager on UARS, J. Geophys. Res., 100, 10,365-10,392, 1995.

Dunkerton, T. J., Theory of the mesopause semiannual oscillation, J. Atmos. Sci., 39, 2681-2690, 1982.

Forbes, J. M., M. Kilpatrick, D. Fritts, A. H. Manson, and R. A. Vincent, Zonal mean and tidal dynamics from space: An empirical examination of aliasing and sampling issues, Ann. Geophys., 15, 1158-1164, 1998.

Garcia, R. R., T. J. Dunkerton, R. S. Lieberman, and R. A. Vincent, Climatology of the semiannual oscillation of the tropical middle atmosphere, J. Geophys. Res., 102, 26,019-26,032, 1997.

Hays, P. B., V. J. Abreu, M. E. Dobbs, D. A. Gell, H. J. Grassl, and W. R. Skinner, The High Resolution Doppler Imager on the Upper Atmosphere Research Satellite, J. Geophys. Res., 98, 10,713-10,723, 1993.

Holton, J. R., The influence of mean wind shear on the propagation of Kelvin waves, Tellus, 22, 186-193, 1970.

Holton, J. R., The influence of gravity wave breaking on the general circulation of the middle atmosphere, J. Atmos. Sci., 40, 2497-2507, 1983.

Holton, J. R. and R. S. Lindzen, A note on "Kelvin" waves in the atmosphere, Mon. Weath. Rev., 96, 385-386, 1968.

Lieberman, R. S. and D. Riggin, High resolution Doppler imager observations of Kelvin waves in the equatorial mesosphere and lower thermosphere, J. Geophys. Res., 102, 26,117-26,130, 1997.

Lieberman, R. S., M. D. Burrage, D. A. Gell, P. B. Hays, A. R. Marshall, D. A. Ortland, W. R. Skinner, and D. L. Wu, Zonal mean winds in the equatorial mesosphere and lower thermosphere observed by the High Resolution Doppler Imager, Geophys. Res. Lett., 20, 2849-2852, 1993.

Lindzen, R. S., Equatorial planetary waves in shear: Part I, J. Atmos. Sci., 28, 609-622, 1971.

Lindzen, R. S., Turbulence and stress owing to gravity wave and tidal break- down, J. Geophys. Res., 86, 9707-9714, 1981.

Lindzen, R. S. and J. R. Holton, A theory of the quasi-biennial oscillation, J. Atmos. Sci., 25, 1095-1107, 1968.

Ortland, D. A., P. B. Hays, W. R. Skinner, M. D. Burrage, A. R. Marshall, and D. A. Gell, A sequential estimation technique for recovering atmospheric data from orbiting satellites, in The Upper Mesosphere and Lower Thermosphere: A Review of Experiment and Theory, Geophys. Monograph Series, vol. 87, edited by R. M. Johnson and T. L. Killeen, pp. 329-337, AGU, Washington, D.C., 1996.

Ortland, D. A., P. B. Hays, W. R. Skinner, and J.-H. Yee, Remote sensing of mesospheric temperature and $\mathrm{O}_{2}\left({ }^{1} \Sigma\right)$ band volume emission rates with the high resolution Doppler imager, J. Geophys. Res., 103, 1821-1835, 1998.

Randel, W. J., The evaluation of winds from geopotential height data in the stratosphere, J. Atmos. Sci., 44, 3097-3120, 1987.

Sassi, F. and R. R. Garcia, A one-dimensional model of the semiannual oscillation driven by convectively forced gravity waves, J. Atmos. Sci., 51, 3167-3182, 1994.

Sassi, F. and R. R. Garcia, The role of equatorial waves forced by convection in the tropical semiannual oscillation, J. Atmos. Sci., 54, 1925-1942, 1997.

Smith, A. K., Longitudinal variability of the mesopause SAO, Geophys. Res. Lett., 24, 1991-1994, 1997.

Talaat, E. and R. Lieberman, Nonmigrating diurnal tides in mesospheric and lower thermospheric winds and temperatures, J. Atmos. Sci., 1998 (submitted).

A. K. Smith (e-mail: aksmith@ucar.edu) 